\title{
Human genome variability and host-pathogen interactions Lluis Quintana-Murci
}

Address: Institut Pasteur/CNRS, Paris, France

from Fifth Dominique Dormont International Conference. Mother-to-child transmitted viral diseases: from transmission to children care

Paris, France. 26-28 March 2009

Published: 22 July 2009

Retrovirology 2009, 6(SuppI I):LI doi:I0.II86/1742-4690-6-SI-LI

This abstract is available from: http://www.retrovirology.com/content/6/SI/LI

(C) 2009 Quintana-Murci; licensee BioMed Central Ltd.

Natural selection is a major force behind the shaping of patterns of human genome variability. Inferences concerning the action of selection in the human genome provide a powerful tool for predicting regions of the genome of major functional importance. Genetic variants influencing human susceptibility to disease are likely to affect the fitness of the organism, unless the disease concerned begins late in the life. There is therefore an intimate relationship between disease and selection that can be exploited for the identification of candidate disease loci. To date, some of the strongest evidence for selection in the human genome has been obtained for genes involved in the immune response or host-pathogen interactions. Indeed, before the advent of antibiotics and vaccines, infectious diseases have been paramount among the threats to health and survival for most of human evolutionary history. I will review our most recent studies searching for the footprints of natural selection in the human genome. These studies, which go from global genomewide scans to more fine-tuned analyses in specific genes, highlight how the identification of selected loci or variants may provide insight into host genes or pathways playing an important role in pathogen resistance. For example, we have shown that natural selection has significantly driven the processes of population differentiation in modern human populations. Specifically, we have identified a number of genes under strong geographicallyrestricted positive selection, some of them involved in host-pathogen interactions. In addition, I will present our most recent data on the Toll-like receptor (TLR) signalling pathway. Our evolutionary data indicate that the different members of TLR family differ in their ecological relevance and increase our understanding of how variation in these genes results in different contributions to the outcome of infectious diseases. More generally, I will show how the identification of the extent and type of selection acting upon human genes involved in host-pathogen interac- tions make it possible to define the redundant and nonredundant functions of individual immunity-related genes in the natural setting. 